\title{
Application of the duality in the finite element analysis of shell: self-dual metis planar shell elements
}

Nguyen Dang Hung ${ }^{1,2}$

Correspondence: hung.nd@vgu.edu.vn ${ }^{1}$ Vietnamese-German University, Binh Duong New City, Vietnam University of Liège, Liège B4000, Belgium

\section{Springer}

\begin{abstract}
In this paper, an application of the duality principle in finite element shell analysis is presented. It is based upon Lure (Prikl. Mat. Mekh, XIV 5, 1958) and Goldenveizer's (Theory of thin shells, Pergamon Press, 1961) theory of thin shells. For the finite element analysis of thin shells, using the stress function, the problem of finding a stress fields in equilibrium and expressing their continuity across the interfaces is shown to be identical to the problem of derivation of conforming displacement fields and vice versa. It appears that one may derive from this concept of duality a new family of shell finite elements: the auto-dual shell elements. In this paper, we consider a self-dual flat element for thin shell analysis. This element is based on the already developed and implemented in LTAS department, University of Liège. It was mixed hybrid elements called 'mixed métis'. Numerical efficiency is demonstrated by means of some examples: cylindrical shell roof simply supported by two diaphragms and submitted to its dead weight and cylinder loaded by two diametrically opposed point loads, clamped hyperbolic shell loaded by uniform normal pressure.
\end{abstract}

Keywords: Thin shell finite element; Hybrid and Métis Model; Duality in shell analysis; Stress and displacement element; Equilibrium element; Stress function

\section{Background}

Expanding from the analogy between flexure and extension of flat plates [1], Fraeys De Veubeke and Zienkiewicz [2] and, later, Elias Ziad [3] have pointed out the possibility of dual analysis of plates using equilibrium and conforming finite elements. The generalization of this duality to the case of curved shell from the finite element point of view has been presented by Nguyen Dang Hung [4]. These considerations are based on the static-geometric analogy due to Lure [5] and Goldenveizer [6]. In this paper, we discuss a further development of this duality by combining a hybrid stress and displacement model into a flat shell element.

This hybridmixed planar shell element is self-dual because the shape functions of the dual quantities (Airy's stress function for the membrane effect and the vertical deflection for the bending effect, on one hand, stretching displacement field and bending stress-function, on the other hand) are the same. The choice of the nodal connections is such that nodal displacements or mean displacements are the unknowns: the 
element can therefore be readily implemented into finite element algorithms based upon the displacement method.

Both the bending element and the membrane element which combine into the planar shell element belong to a special class of hybrid elements called 'metis element' $[7,8]$. The important advantages exhibited by these elements concerning speed of convergence and stability in stress calculation are expected in the present case.

A raw version about the existence of this element was presented long time ago in Madrid [9] but this present version is more detailed mostly about the detailed illustrations of the concept of self-dual finite elements.

\section{Methods}

\section{Development of the element}

\section{Duality in the finite element analysis of shells}

Lure [5] and Goldenveizer [6] have demonstrated that a perfect analogy does exist between the stress quantities and the strain quantities in the formulation of thin shell theory. Compatibility equations for strains and displacement components become equilibrium equations for stresses and stress function components when dual quantities are replaced by each other as follows:

$$
\begin{aligned}
& \left|u, v, \mathrm{w}, \varepsilon_{1}, \varepsilon_{2}, \omega_{12}, \omega_{21}, \kappa_{1}, \kappa_{2}, \tau_{12}, \tau_{21}\right| \\
& \left|U, V, W,-M_{2},-M_{1}, M_{12}, M_{21}, N_{2}, N_{1},-N_{12}\right|
\end{aligned}
$$

Where $d^{T}=|u, v, w|$ are the displacement components defined on the middle surface

$$
\mathbf{a}^{T}=\left|-M_{2},-M_{1}, M_{12}, M_{21}, N_{2}, N_{1},-N_{21},-N_{12}\right|
$$

are the moments and membrane forces defined per unit length of middle surface as in the classical shell theory, see for example reference [10], $U, V$, and $W$ are the stress functions.

It is shown that equilibrium and compatibility are exactly satisfied with the following definition of the strain and stress:

$$
\varepsilon=\nabla d, \sigma=\nabla D
$$

where $\mathbf{D}^{\mathrm{T}}=|U, V, W|$ are the stress function components defined on the middle surface

$$
\begin{aligned}
& \tilde{N}^{\mathrm{T}} \text { a derivative operator of matrix form [3x8] } \\
& \mathrm{a} 1=\frac{1}{2 R_{1}}\left(\frac{\partial}{A_{2} \partial \alpha_{2}}-\frac{1}{\rho_{1}}\right) ; \delta_{1}=\frac{\partial}{A_{1} \partial \alpha_{1}}\left(\frac{1}{R_{1}}\right)+\frac{1}{2}\left(\frac{1}{R_{1}}+\frac{1}{R_{2}}\right) \frac{\partial}{A_{1} \partial \alpha_{1}}+\frac{1}{2 R_{1} R_{2}}\left(R_{1} \frac{\partial}{A_{1} \partial \alpha_{1}}-\frac{R_{2}}{\rho_{2}}\right) \\
& \mathrm{a}_{3}=-\frac{\partial}{A_{1} \partial \alpha_{1} A_{2} \partial \alpha_{2}}+\frac{\partial}{\rho_{1} A_{1} \partial \alpha_{1}} ; \delta_{2}=\frac{\partial}{A_{2} \partial \alpha_{2}}\left(\frac{1}{R_{2}}\right)+\frac{1}{2}\left(\frac{1}{R_{1}}+\frac{1}{R_{2}}\right) \frac{\partial}{A_{2} \partial \alpha_{2}}+\frac{1}{2 R_{1} R_{2}}\left(R_{2} \frac{\partial}{A_{2} \partial \alpha_{2}}-\frac{R_{1}}{\rho_{1}}\right) \\
& \mathrm{b}_{2}=\frac{1}{2 R_{2}}\left(\frac{\partial}{A_{1} \partial \alpha_{1}}-\frac{1}{\rho_{2}}\right) ; \mathrm{b}_{3}=-\frac{\partial^{2}}{A_{2} \partial \alpha_{2} A_{1} \partial \alpha_{1}}+\frac{\partial}{\rho_{2} A_{2} \partial \alpha_{2}}
\end{aligned}
$$

$\alpha_{1}, \alpha_{2}$, orthogonal coordinate system

$A_{1}, A_{2}$, corresponding Lame's system

$\rho_{1}, \rho_{2}$, radius of geodesic curvature on the middle surface 


\section{Conformity and diffusivity}

A displacement field satisfies conformity in a curved shell if the following continuity is insured along any edge of the shell element:

$$
\left(u_{n}\right)^{+}=\left(u_{n}\right)^{-},\left(u_{n}\right)^{+}=\left(u_{s}\right)^{-},\left(w_{n}\right)^{+}=\left(w_{n}\right)^{-},\left(\frac{\partial w}{\partial n}\right)^{+}=\left(\frac{\partial w}{\partial n}\right)^{-}
$$

Where $\vec{n}$ and $\vec{s}$ are the middle surface normal and tangent to the edge, respectively. A stress resultant field satisfies diffusivity if the following quantities are continuously transmitted through the boundary of the element.

$$
\begin{aligned}
& \left(N_{n}\right)^{+}=\left(N_{n}\right)^{-},\left(\bar{N}_{s}\right)^{+}=\left(N_{s}+M_{n} s / R_{s}\right)^{+}=\left(\bar{N}_{s}\right)^{-},\left(M_{n}\right)^{+}=\left(M_{n}\right)^{-} \\
& \left(K_{n}\right)^{+}=Q_{n}+\left(? M_{n} s\right) / ? s=\left(K_{n}\right)^{-}
\end{aligned}
$$

And the local jumps of the twisting moment at vertex $\mathrm{k}$ is as follows:

$$
Z_{k}=\left(M_{n} s\right)_{k}^{+}-\left(M_{n} s\right)_{k}^{-}
$$

Nguyen Dang Hung [4] has presented a boundary duality theorem which states that: 'If displacements conformity is satisfied, stress resultants diffusivity is also satisfied when the same fields are used for displacement and stress functions'. In other words, let us choose a shape function for the displacement field $d$, making use of some appropriate connectors (nodal displacements) on the boundary, such that (5) is satisfied; if the same shape function is chosen for the stress function field $\mathrm{D}$, that is similar assumptions on the field and corresponding connectors (nodal stress functions), then equilibrium conditions on the boundary (6) are automatically satisfied.

In the case of planar shell $\left(\frac{1}{R_{1}}=\frac{1}{R_{2}}=0\right)$, there is no coupling between membrane stress components and bending moments and if Cartesian coordinates are used $\left(\frac{1}{\rho_{1}}=\frac{1}{\rho_{2}}=0\right)$, the derivative operator $\nabla$ is reduced to a simpler form:

$$
\nabla^{T}=\left[\begin{array}{cccccc}
\frac{\partial}{\partial x} & 0 & \frac{\partial}{2 \partial y} & 0 & 0 & 0 \\
0 & \frac{\partial}{\partial y} & \frac{\partial}{2 \partial x} & 0 & 0 & 0 \\
0 & 0 & 0 & \frac{\partial^{2}}{\partial x^{2}} & \frac{\partial^{2}}{\partial y^{2}} & -2 \frac{\partial^{2}}{\partial x \partial y}
\end{array}\right]
$$

The dual quantities become

$$
\begin{aligned}
& d^{T}=|u, v, \mathrm{w}| ; \varepsilon^{T}=\left|\varepsilon_{x}, \varepsilon_{y}, \frac{\gamma_{x y}}{2}, \kappa_{x}, \kappa_{y}, \kappa_{x y}\right| \\
& D^{T}=|U, V,-F| ; \sigma^{T}=\left|-M_{y},-M_{x}, M_{x y}, N_{y}, N_{x},-N_{x y}\right|
\end{aligned}
$$

Where we recognize $U$ and $V$ as Southwell's stress functions (for bending effects) and $F$ as Airy's stress function (for the membrane part). The boundary duality theorem identifies the problem of finding stress functions $U$ and $V$ and of expressing their 
continuity across the interface with the problem encountered in the derivation of conforming displacement fields for membrane stretching [11]:

$(\mathrm{u}, \mathrm{v})$ continuous entails $(\mathrm{U}, \mathrm{V})$ continuous i.e. force components $\mathrm{M}_{\mathrm{n}}, \mathrm{K}_{\mathrm{n}}$ continuous and $Z_{k}=0$ at the vertex $k$.

Conversely, according to the same theorem, the problem of finding a continuous stress field $(N x, N y, N x y)$ and expressing the continuity of the components (Nn, Nns) across the interface of a membrane element is the same as the problems of finding a conforming transversal displacement in plate bending.

$$
\left(N_{n}, N_{n s}\right)\left(F,-\frac{\partial F}{\partial n}\right)\left(w, \frac{\partial w}{\partial n}\right) \text { Continuous }
$$

Figure 1a presents a triangular element with quadratic displacement field along the edges; 12 nodal displacements are necessary to satisfy the conformity.

Figure 1b shows a bent plate finite element with 12 nodal stress functions as connectors; this triangle ensures continuity of the normal moment $M n$ (linear along an edge), the equivalent shear force $K n$ (constant along an edge) and the local jumps of twisting moment $Z k$ at each corner. If forces are taken as nodal values rather than stress functions, the corresponding 12 connectors are those exhibited in Figure 1c.

This system of nodal forces is adopted in reference [12] for the formulation of an equilibrium model for plate bending with linear assumptions for the moment field.

Figure 2a represents a conforming element for plate bending with quadratic assumptions on the vertical deflection $w$ along the edges.

Figure $2 \mathrm{~b}$ shows the dual membrane element with nodal values of the Airy' stress function; it is equivalent to the element shown on Figure $2 \mathrm{c}$ where the resultants.

$R_{x}=\int\left(l \cdot N_{x}+m \cdot N_{x y}\right) d x ; \quad R_{y}=\int\left(m . N_{y}+l \cdot N_{x y}\right) d y$ have been chosen as nodal values.

$(l, m$ are the components of the unit normal to the edge).

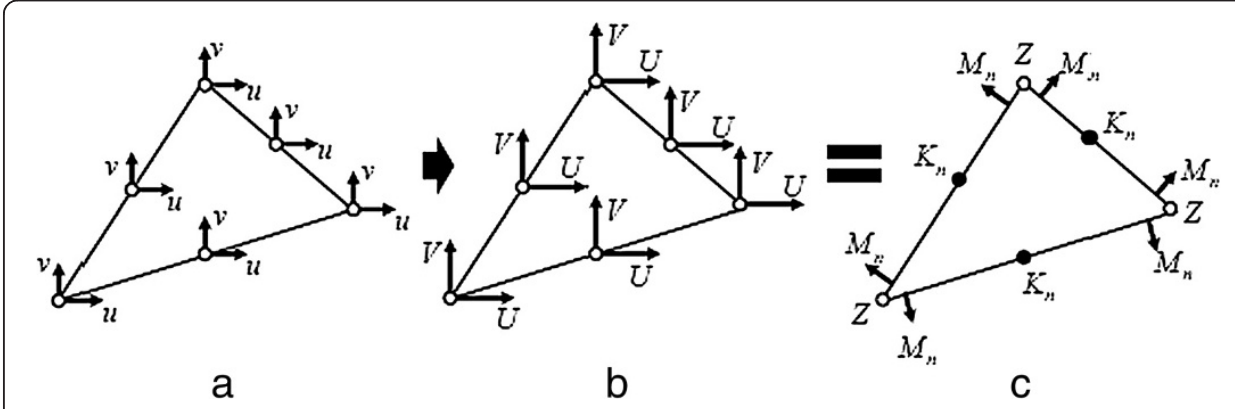

Figure 1 A triangular element with quadratic displacement field along the edges. (a) Membrane element with quadratic displacement field. Conformity is satisfied with 12 nodal connectors. (b) Dual element for plate bending with quadratic stress field. Boundary equilibrium (diffusivity) is satisfied with 12 stress connectors. (c) Corresponding element for plate bending with linear moment field. Boundary equilibrium (diffusivity) requires 12 nodal forces. 


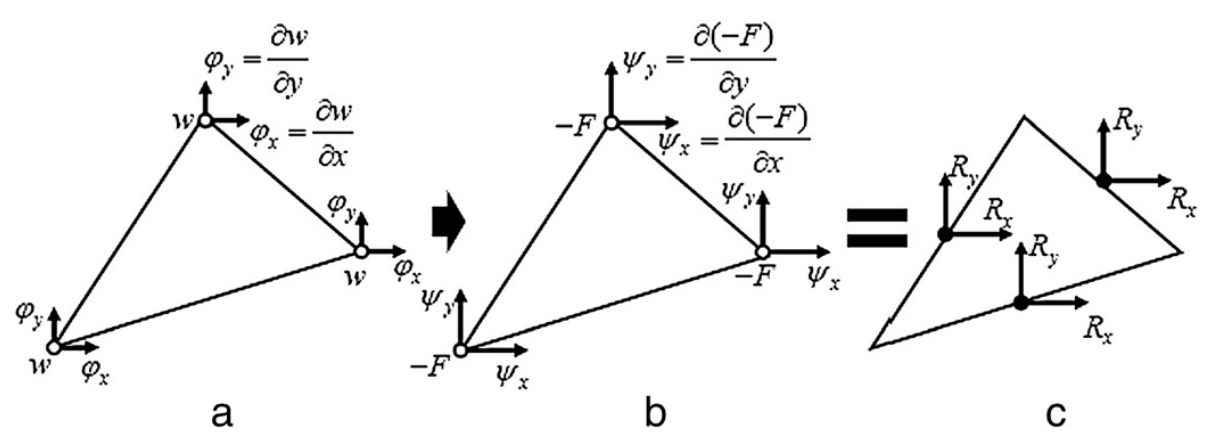

Figure 2 Elements of plate bending and membrane stretching. (a) Element of plate bending with quadratic deflection. Conformity requires 2 nodal displacements on each edge. (b) Dual element for membrane stretching with quadratic Airy's function. Diffusivity requires nine nodal stress function connectors. (c) Corresponding element for membrane stretching constant stress field. Boundary equilibrium (diffusivity) requires six nodal forces.

\section{Hybrid finite elements and métis finite elements}

Let us now consider the following mixed hybrid functional:

$$
I=I_{T}+I_{B}
$$

Where $I T$ is the modified complementary energy functional for the membrane effect:

$$
\left.I_{T}=\sum_{N}\left(\int_{A N} \frac{1}{2} T_{i j k l} \cdot N_{i j} N_{k l}\right) d A-\int_{\Gamma_{N}} n_{j} \cdot N_{i j} \tilde{u_{i}} d s+\int_{\Gamma_{\sigma N}} n j \cdot \bar{N}_{i j} \tilde{u_{i}} d s\right)
$$

and $I B$ is the modified potential energy functional for the bending effect:

$$
\begin{aligned}
I_{B}= & \sum_{N}\left(\int_{A_{N}}\left(\frac{1}{2} B i_{j k l} \cdot K_{i j} K_{k l}-p w\right) d A+\int \Gamma u_{N}\left[M_{n} \sim \frac{\partial \bar{w}}{\partial n}-\widetilde{K_{n}} \bar{w}+\frac{\partial}{\partial s}\left(\widetilde{M_{n s}} \bar{w}\right)\right] d s\right. \\
& \left.-\int_{\Gamma_{N}}\left[M_{n} \frac{\partial w}{a n}-\widetilde{K_{n}} w+\frac{\partial}{\partial s}\left(M_{n s} w\right)\right] d s\right)
\end{aligned}
$$

In these expressions,

$$
\begin{aligned}
T i_{j k l} & =[T]=\frac{1}{E h}\left[\begin{array}{ccc}
1 & -v & 0 \\
-v & 1 & 0 \\
0 & 0 & 2(1+v)
\end{array}\right] \text { is the elastic compliance of the stretching effect } \\
B_{i j k l} & =[B] \\
& =\frac{E h^{3}}{12\left(1-v^{2}\right)}\left[\begin{array}{ccc}
1 & v & 0 \\
v & 1 & 0 \\
0 & 0 & 2(1-v)
\end{array}\right] \text { is the elastic compliance of the bending effect }
\end{aligned}
$$

$A N$, the domain of element

$N$, its boundary

$\Gamma_{\sigma_{N}}$, portion of where tensions $n_{j} \bar{N}_{i j}=\bar{T}_{i}$ are prescribed

$p$, normal pressure

$h$, thickness of the shell 
One may notice that in (13) there are two unknown fields: the stress field $N i j$ must be defined and in equilibrium over the domain $A N$.

The displacement field $\tilde{u}_{i}$ is defined on the boundary $\Gamma_{N}$ in such a way that conformity (i.e., the two first relations of 5) is insured. This functional was first proposed by Pian $[13,14]$ for a hybrid stress finite element formulation. On the other hand, functional (14) possesses two other unknown fields: the deflection $w$ must be defined continuously in $A N$; the stress field $\left[\begin{array}{lll}\tilde{M}_{n} & \tilde{K}_{n} & \tilde{Z}\end{array}\right]$ must be defined on the boundary $\Gamma_{N}$ in such a way that diffusivity (i.e., three last relations of 6 ) is satisfied.

This functional is adopted by Jones [15] for a hybrid displacement finite element formulation.

Nguyen Dang Hung $[7,8]$ has shown that if the boundary conforming field $\tilde{u}_{i}$ of (13) defined on the boundary $\Gamma_{N}$ can be extended over $A N$ in other words if the two unknown fields in (13) are well defined everywhere in the closed domain $\bar{A}_{N}$, the hybrid stress element belongs to a special class called 'mongrel displacement element' which leads to important advantage in energy convergence. In the same way, if the two unknown fields of functional (14) are well defined in the closed domain $\bar{A}_{N}$ (i.e., equilibrium boundary field $\left[\begin{array}{lll}\tilde{M}_{n} & \tilde{K}_{n} & \tilde{Z}\end{array}\right]$ can be extended everywhere in $\bar{A}_{N}$ ), the corresponding hybrid displacement element for plate bending becomes a 'mongrel stress element' with the same properties concerning the convergence.

In this paper, such is the case for the mixed hybrid planar shell element described in this paper as well for membrane as for bending effects.

\section{Self-dual metis planar shell element}

Let us make the following assumptions concerning the four unknown fields of the hybrid mixed functional (12):

$$
\begin{aligned}
& w=\sum_{m=0}^{M} \sum_{n=0}^{n} \beta_{m n} x^{m-n} y^{n} ;-F=\sum_{m=0}^{M} \sum_{n=0}^{n} \beta_{m n}^{\prime} x^{m-n} y^{n} \\
& \widetilde{\sim}=\alpha_{1}+\alpha_{2} x+\alpha_{3} y+\alpha_{4} x^{2}+\alpha_{5} x y+\alpha_{6} y^{2} \\
& \widetilde{\sim}=\alpha_{7}+\alpha_{8} x+\alpha_{9} y+\alpha_{10} x^{2}+\alpha_{11} x y+\alpha_{12} y^{2} \\
& V^{\sim}=\alpha_{7}^{\prime}+\alpha_{8}^{\prime} x+\alpha_{9}^{\prime} y+\alpha_{10}^{\prime} x^{2}+\alpha_{11}^{\prime} x y+\alpha_{12}^{\prime} y^{2} \\
& \widetilde{U^{\sim}}=\alpha_{1}^{\prime}+\alpha_{2}^{\prime} x+\alpha_{3}^{\prime} y+\alpha_{4}^{\prime} x^{2}+\alpha_{5}^{\prime} x y+\alpha_{6}^{\prime} y^{2}
\end{aligned}
$$

Where $\beta_{m n}, \beta^{\prime}{ }_{m n}, \alpha_{i}, \alpha^{\prime}{ }_{i}$ are the interpolation parameters,

$M$ is the maximum degree of the polynomial (15).

Let us adopt for the membrane element the natural system of nodal displacements shown on Figure 1a and for the bending effect the natural system of nodal stress functions shown on Figure 1b. It appears that we will have a mongrel-mixed planar shell element. Assumptions (16) indicate that the boundary fields $\left(\begin{array}{ll}\tilde{u} & \tilde{v}\end{array}\right)$ and $\left(\begin{array}{lll}\tilde{U} & \tilde{V}\end{array}\right)$ are defined everywhere in $\bar{A}_{N}$ and conformity and diffusivity are both satisfied with the system of 24 nodal values shown on Figure 3.

Dual quantity $<w>$ is a sort of mean vertical deflection of the shell.

This element, denominated 'HYTCOQ' constitutes a self-dual metis planar shell element because the strain field and the stress field for the membrane and the bending effects are respectively dual quantities of each other in the sense discussed in the section Duality in the finite element analysis of shells. The details of the stiffness 


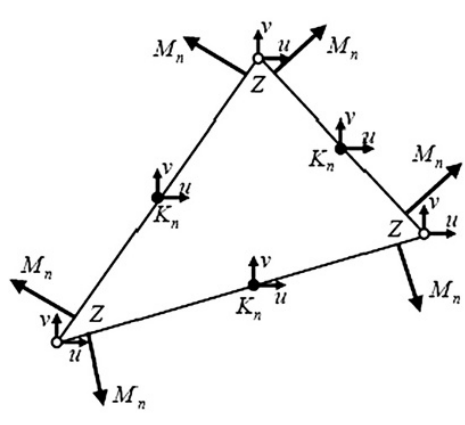

a

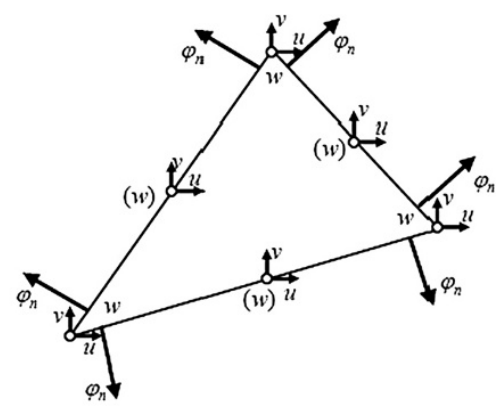

b

Figure 3 Mixed and self-dual planar shell element. (a) Metis mixed planar shell element. This element is self-dual because its bending and stretching effect are represented by dual models of Figure 1a,c. (b) Self-dual metis mixed planar shell element (HYTCOQ). The nodal forces $\left[\begin{array}{lll}\tilde{M}_{n} & \tilde{K}_{n} & \tilde{Z}\end{array}\right]$ are replaced by the nodal displacement generalized one $\left[\begin{array}{lll}\varphi_{n} & \langle w\rangle & w\end{array}\right]$.

matrix formation for the membrane and bending effects are respectively given in references [16,17].

Here, we merely observe that, for the bending effect, we have formulated the element in such a way that nodal generalized displacements replace nodal forces as unknowns; in this way, no special modifications are required to run this element on existing codes written for displacement elements.

On the other hand, we notice that the system of nodal displacements of this element (Figure $3 \mathrm{~b}$ ) is well suited for easy connection of adjacent elements. In particular, the normal slope is locally defined on the edge of the element; this avoids the drawback frequently encountered with flat shell elements when the slopes are defined at the corners.

One may summarize here the nature of HYTCOQ:

(a) The membrane part possesses displacement metis stretching element with quadratic displacement field defined on the vertexes. The equilibrium stress field (which is derived from a polynomial Airy's function) is defined only inside the element. It appears that when the degree of the Airy's function such that at least $M=4$, stress field is quadratic the normal rule for kinematic stability is respected. In these conditions, the displacement hybrid or metis formulation does not imply spurious modes, this element leads to good behavior in convergence and precision according to the numerical tests realized in LTAS. Recently (2013), a new examination is performed and it appears that this element leads to very good performance in terms of convergence, precision, and numerical stability [18].

(b) The bending part possesses stress metis element with linear moment field defined on the vertexes. The vertical defection (which is derived from a polynomial function) is defined only inside the element. This bending stress metis element was examined intensively, and the very good results are described in the paper [17].

As both stretching and bending effects are represented by very good elements and there exists no interaction effect due to the flat geometry, we must expect to a good performance of the present self-dual planar shell element. 


\section{Results and discussion \\ Numerical results \\ Preliminary remarks}

This section intends to show the performances of this new mongrel-mixed element by means of a few examples. Simply or doubly curved shells are chose as, namely, cylindrical roof and cylinder or hyperbolic roof. Each example is illustrated by several diagrams. On each of these:

- A small drawing represents the structure, its boundary conditions, its loading, all pertinent data and the finest discretization of mesh.

- The elements being compared are represented by a symbol; their meaning is defined on every figure because it can vary from one figure to the next; the results of a new element called 'HYTCOQ' will be always illustrated in the figures.

- Appropriate references are also given [19].

- The stresses shown are always those obtained with the finest grid; it was deemed useful to represent these stresses even in case where no analytical solution or other numerical results are available.

\section{Cylindrical shell roof supported by two vertical diaphragms and loaded by its dead weight}

It is useful to mention that the vertical diaphragms restrain the displacement along $X$ and $Z$ directions but let free $Y$ displacement. The rotation in the direction of $X$ axis is not prevented. The double symmetry of the structure allows considering only a quarter of the structure.

Figure 4 shows the convergence of the strain energy. The analytical solution is taken from Scordelis and Lo [20]. The HYTCOQ element exhibits a relatively fast convergence, and the exact solution is reached with less than 300 degrees of freedom. For comparison motivation, we have chosen the 'GSS3' curved triangular element elaborated by Idelsohn [21]. This latter has really a better behavior than HYTCOQ possibly because the curvature is taken into account.

We can finally notice the following important fact: the convergence of the strain energy seems to be monotonous and upper bounds are obtained for this example; we will see that this monotony is preserved for other examples even if lower bounds are obtained. This remarkable situation has been theoretically discussed by Nguyen Dang Hung $[7,8]$.

Figure 5 illustrates the convergence of the displacement at the point $\mathrm{B}$. The analytical solution has been calculated by Scordelis and Lo [20] and is equal to $6.308 \mathrm{ft}$.

In this example, only the GSS3 has a better behavior than the HYTCOQ.

MTS2 element behaves exactly like HYTCOQ but the others are rather worse although they are curved and therefore well suited for this example.

Note that the analytical solution is about $3 \%$ higher than the 'exact' one towards which elements MTS2 and HYTCOQ converge.

Figure 6 presents bending moments along $\mathrm{AB}$. The finite element stresses are evaluated by calculating a mean value over each element compared with the analytical solution, the results of HYTCOQ with 515 degrees of freedom look excellent: nearly all the points are on the analytical curves. 

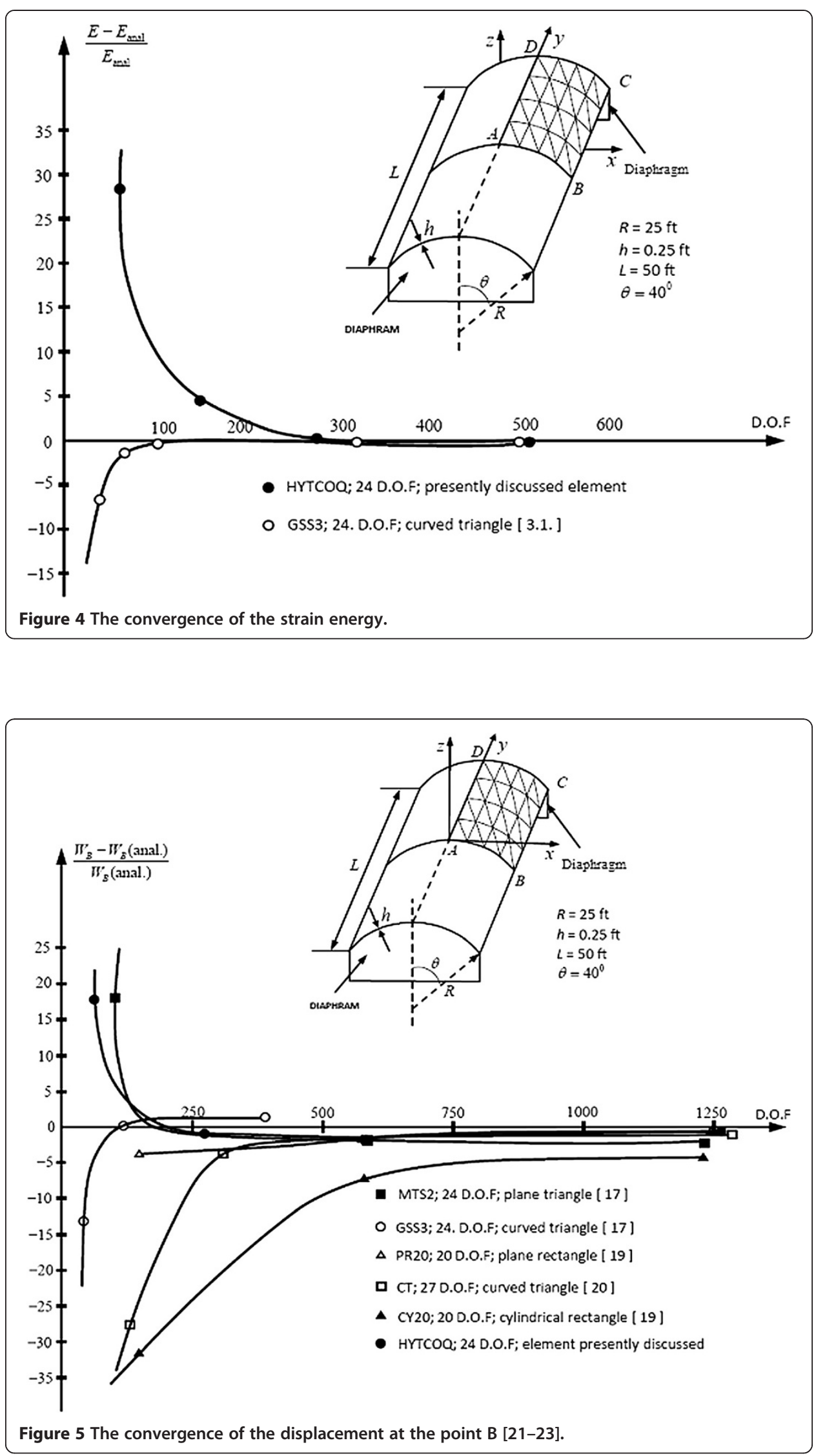


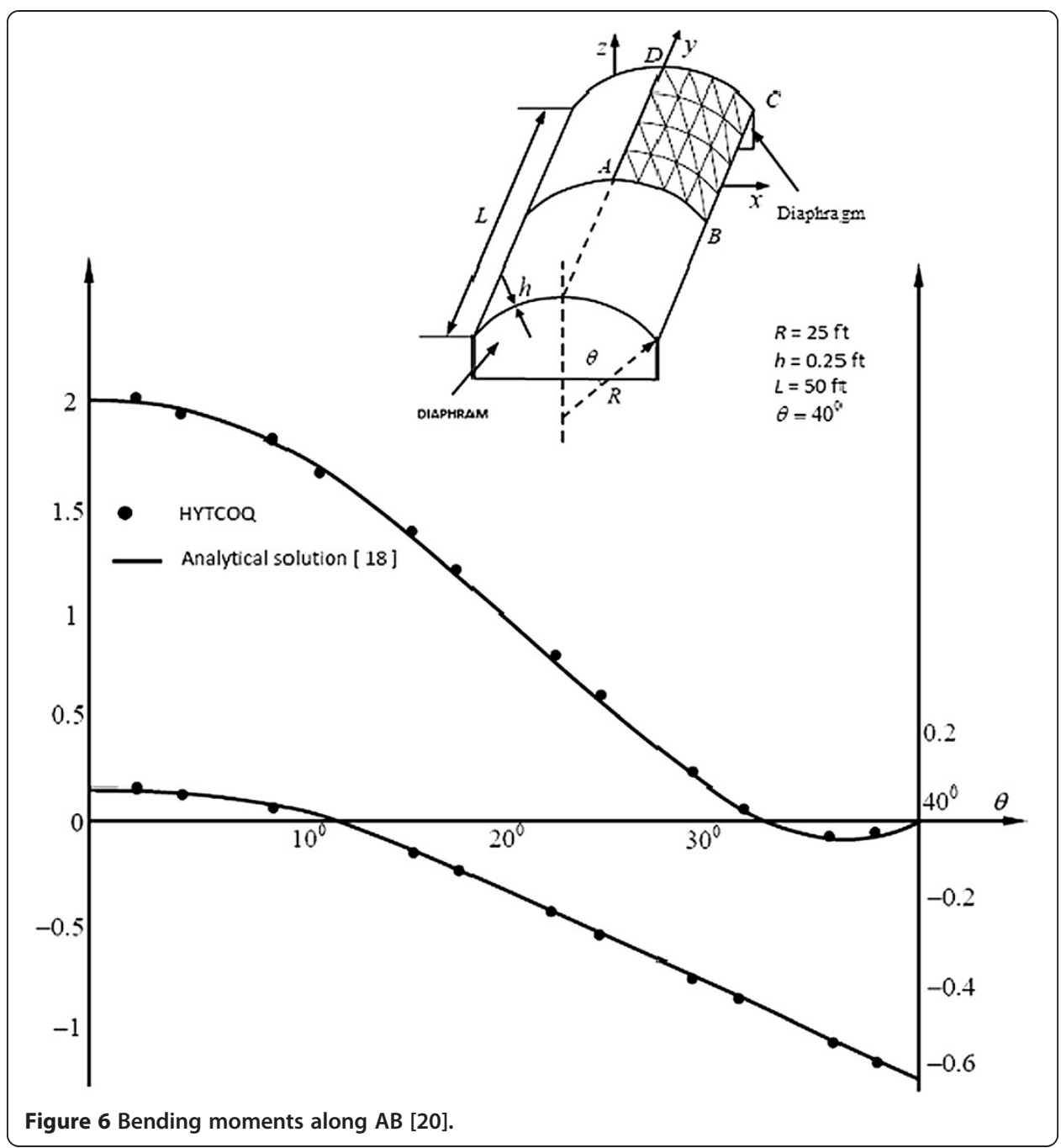

\section{Cylindrical shell}

Because of the symmetry, an eighth of the structure is discretized using HYTCOQ.

Figure 7 presents the convergence of the displacement under the load. We can see that the present element has a very good behavior in comparison with cylindrical elements which are theoretically better suited for this problem.

On this diagram, we can also observe that the behavior of the 'CS' element depends on the type of discretization for there are two different curves for this element; it appears better to choose a given discretization along the curvature than along the axis of the cylinder.

The 'exact' solution towards which all the numerical results do converge seems to be $5 \%$ higher than the analytical solution given by Timoshenko [26]; but this latter includes only bending effects.

Figure 8 illustrates the bending and the membrane stresses along at $y=\mathrm{L} / 2$.

No stress diagrams were found in the literature for this example, but all the stresses tend towards an infinite value under the load except for the Mxy moment which is equal to zero because of symmetry. 

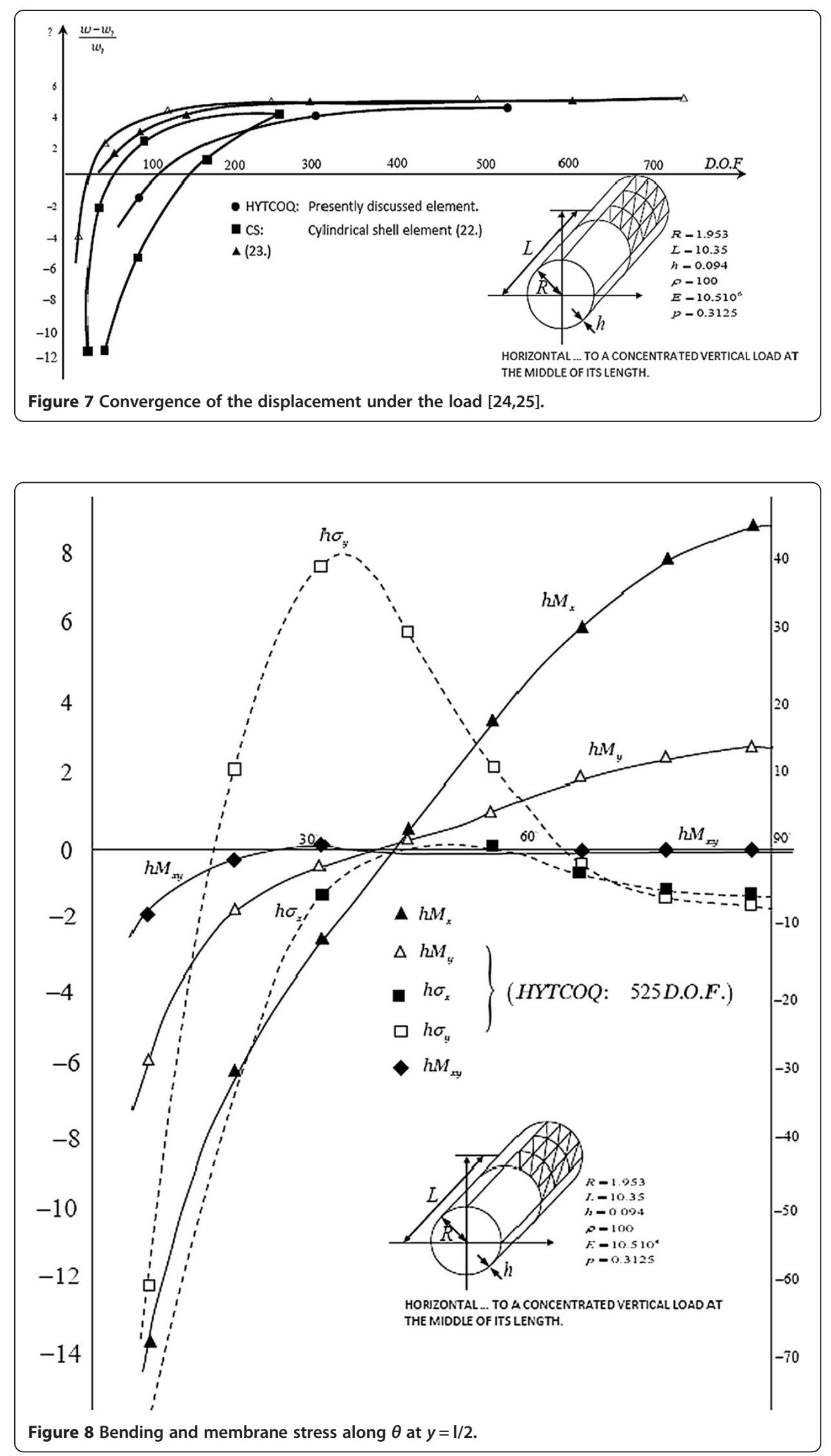


\section{Clamped square hyperbolic parabolic subjected to a uniform normal pressure}

The double symmetry of the structure and the triangular shape of the element allow to study only one quarter of the structure.

Figure 9 presents the convergence of the displacement at the point A. The analytical solution given by Brebbia [27] is equal to $W=$ (where $D$ is represented here the elastic bending rigidity).

The convergence of the HYTCOQ element is not monotonous. This quality is required for only the convergence of the strain energy which indeed does happen (we have not shown it because no reference value was available).

The other elements 'CR20' and 'CR20SE' converge either to another value or more slowly than 'HYTCOQ'.

Figure 10 finally shows the vertical displacement along AD. We compare the result with the result of 'CR20' element. The two solutions are very similar except near the center of the shell, where HYTCOQ gives a better value than CR20 because the exact value obtained from the previous example is $W=-0.02452 \mathrm{~cm}$. Unfortunately, no analytical solution seems to be available to decide which result is best.

This example is interesting because it deals with a doubly curved structure and we can see that the behavior of HYTCOQ element is still very good.

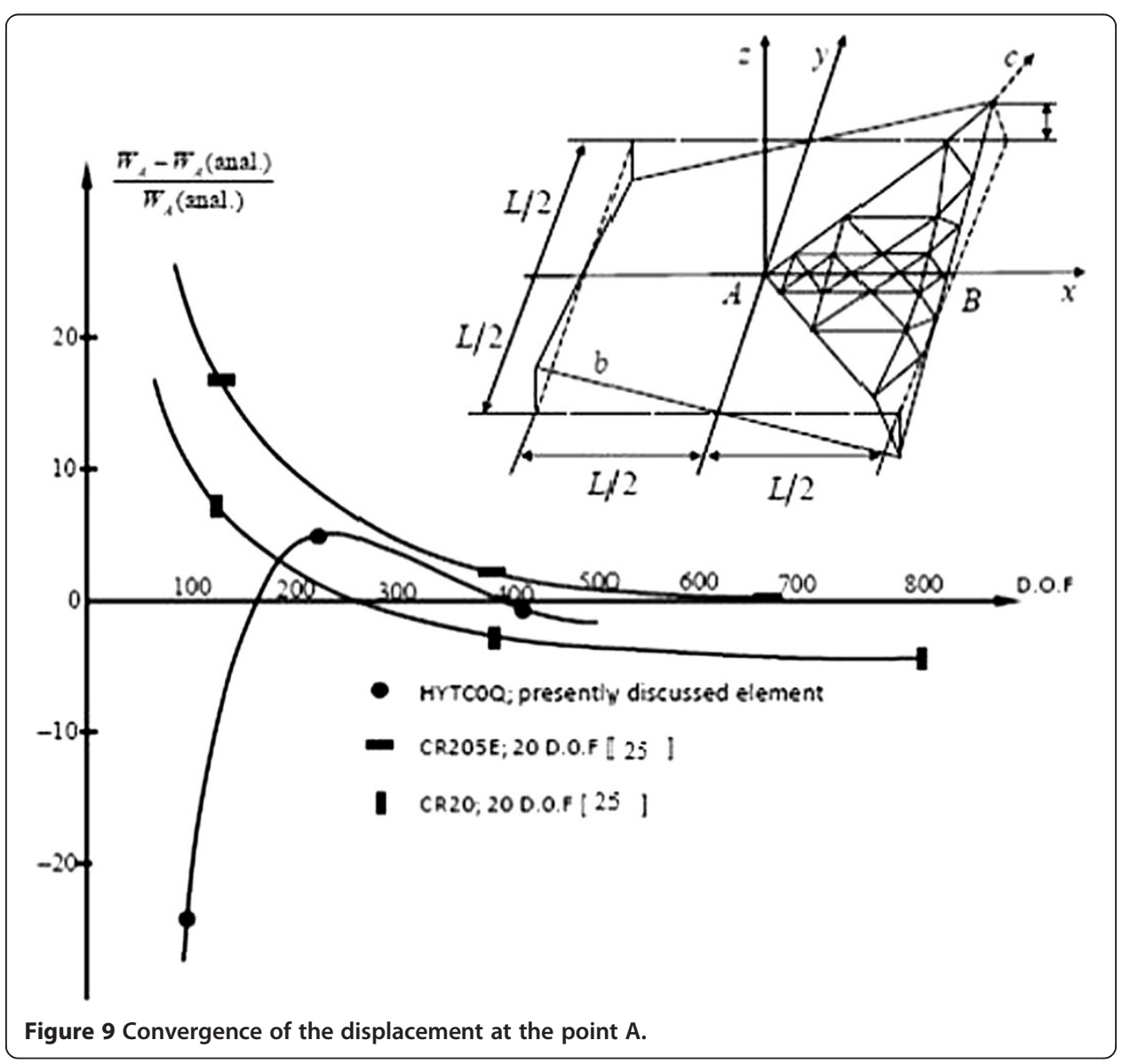




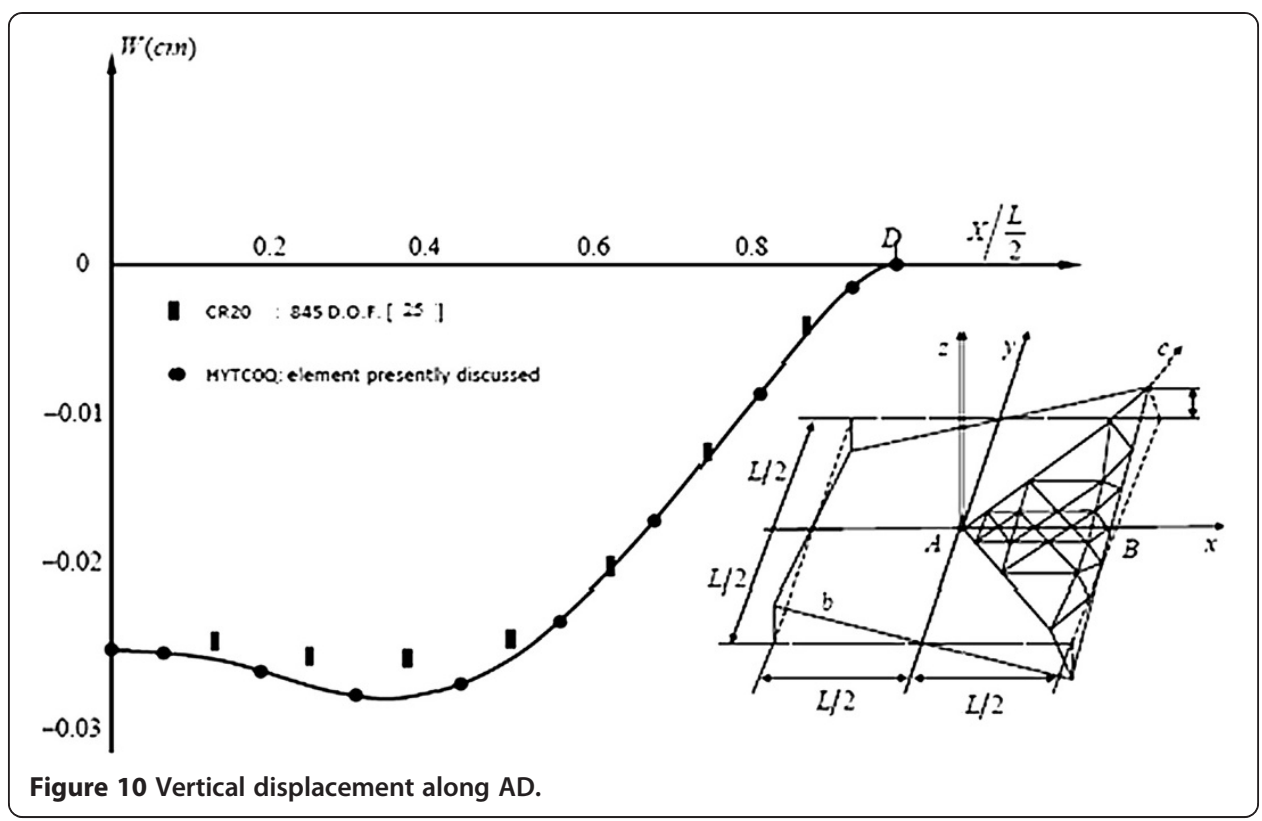

\section{Conclusions}

Implementation of the dual properties in shells is a very powerful tool to generate the appropriate finite elements. In this paper, we focus our attention on a special case: derivation of a self-dual metis planar shell element. The versatility and the fast convergence of this type of finite element are confirmed by numerical experiments. It should be interesting to perform a new self-dual pure planar element composed with the quadratic displacement triangle for stretching aspect and the equilibrium model for plate bending proposed very early by Fraeijs de Veubeke and Sander in 1968. On the other hand, we hope that similar duality in shell analysis should be extended to smooth element method [28].

\section{Competing interests}

The author declares that he has no competing interests.

Received: 5 March 2014 Accepted: 3 June 2014

Published: 12 August 2014

\section{References}

1. Southwell RV (1950) On the analogues relating flexure and extension of flat plates. Quart Mech Appl Math 3:157

2. Fraeijs De Veubeke B, Zienkiewicz OC (1967) Strain-energy bounds in finite element by slab analogy. J Strain Analysis 2(4):265-271

3. Elias Ziad M (1968) Duality in finite element method. J Engrs Mech Div Am Soc Civ Engrs 94(EM4):931-946

4. Hung ND (1971) Duality in the analysis of shells by the finite element method. Int J Solids and Struct 7:281-299

5. Lure Al (1958) The general theory of thin elastic shells. Prikl Mat Mekh XIV:5

6. Goldenveizer AL (1961) Theory of thin shells. Pergamon Press.

7. Nguyen Dang H (1980) On the monotony and the convergence of a special class of hybrid finite elements: the mongrel elements. In: Nemat N (ed) Proceeding of the IUTAM symposium on variational method in the mechanics. Northwestern University, Pergamon Press, pp 208-213

8. Nguyen Dang H (1979) On a special class of hybrid finite elements: the "metis" (in French). In: Proceedings of the 1st International Congress of GAMNI, (E. ABSI and R. GLOWINSKI), Dunod technique, pp 53-63

9. Nguyen Dang H, Detroux P, Falla PH, Fonder G (1979) Proceedings of World Congress on Shell and Spatial Structures, Theme 4. Madrid, pp 4.1-4.19

10. Novozhilov WW (1955) The theory of thin shells translated by JOVE. In: Noordhoff P (ed) Radok JR. The theory of thin shells translated by JOVE. Groningen

11. Sander G (1970) Application of dual analysis principle. In: Proceedings IUTA symposium high speed computing of elastic structures. Liège

12. Fraeijs De Veubeke B, Sander G (1968) An equilibrium model for plate bending. Int J Solids and Structures 4:447-468

13. Pian THH (1964) Derivation of element stiffness matrices by assumed stress distribution. AIAA Jnl 2:1333-1336 
14. Pian $T H H$, Tong $P$ (1969) Basis of finite element methods for solid continua. Int Jnl Num Meth Engr 1:3-28

15. Jones RE (1964) A generalization of the direct stiffness method of structural analysis. AIAA J vol 2, Nr 5:821-826

16. Nguyen Dang H (1976) Direct limit analysis via rigid-plastic finite element. Comp Meth In Mech Engr 8(1):81-116

17. Nguyen Dang H, Desir P (1977) La performance numérique d'un élément fini hybride de déplacement pour l'étude des plaques en flexion » Coll. Publ. Of Faculty of Applied Sciences, vol 33. University of Liège, pp 65-123

18. Dang TD, Hung N-D (2013) A hybrid element model for structural mechanics problems. European Journal of Mechanics - A/Solids 42:469-479

19. Cantin G (1970) Rigid body motions in curved finite elements. AIAA 8(07):1252-1255

20. Scordelis A, Lo KS (1964) Computer analysis of cylindrical shells. J Am Concr Inst 61(5):539-562

21. Idelson S (1974) "Analyse statique et dynamique des coques par la méthodes des éléments finis" Thèse de doctorat ULG

22. Megard G (1969) Planar and curved shell elements. Finite Element Methods in Stress Analysis, Tapir, Trondheim

23. Bunnes G, Dhatt G, Giroux Y, Robichaud L (1968) Curved triangular elements for analysis of shells. In: Proc. 2nd Air Force Conf. on Matrix Methods in Structural Mechanics. Wright-Paterson Air Force base, AFFDL, Ohio, pp 68-150

24. Altman W, lguti $F(1976)$ A thin cylindrical shell element based on a mixed formulation. Computers \& Structures 6(2):149-155

25. Ashwell DG, Sabir AB (1971) A new cylindrical shell finite element based on simple independent strain functions. Department of Civil Engineering, Report, University of Wales, Cardiff

26. Timoshenko SP, Winowsky-Krieger S (1959) Theory of plates and shells. Mc Graw-Hill, New York

27. Brebbia C (1967) Hyperbolic paraboloidal shells, Ph D Thesis. University of Southampton, Department of Civil Engineering

28. Nguyen-Thanh N, Rabczuk T, Nguyen-Xuan H, Bordas S (2008) A smoothed finite element method for shell analysis. Comput Methods Appl Mech Eng 198:165-366

doi:10.1186/s40540-014-0011-6

Cite this article as: Hung: Application of the duality in the finite element analysis of shell: self-dual metis planar shell elements. Asia Pacific Journal on Computational Engineering 2014 1:11.

\section{Submit your manuscript to a SpringerOpen ${ }^{\circ}$ journal and benefit from:}

- Convenient online submission

Rigorous peer review

- Immediate publication on acceptance

- Open access: articles freely available online

- High visibility within the field

Retaining the copyright to your article

Submit your next manuscript at $\boldsymbol{~ s p r i n g e r o p e n . c o m ~}$ 